\title{
EL NEOLÍTICO EN LA ALTA ANDALUCÍA: CUESTIONES SOBRE LA CARACTERIZACIÓN DE SUS FASES
}

\section{THE NEOLITHIC IN UPPER ANDALUSIA: QUESTIONS ABOUT THE CHARACTERIZATION OF HIS PHASES}

\author{
por \\ Beatriz Gavilán Ceballos y \\ J. CARLos Vera Rodríguez
}

RESUMEN En este artículo no pretendemos analizar todas y cada una de las periodizaciones y de las distintas fases que se han propuesto para parcelar el devenir del Neolítico andaluz, sino comentar determinados aspectos relacionados con los criterios de caracterización empleados en recientes propuestas de periodización tripartita, que creemos pueden ser objeto de importantes matizaciones, sobre todo en relación con la secuencia estratigráfica últimamente obtenida en la Cueva de los Murciélagos de Zuheros. Si bien una división en tres fases (Antiguo, Medio y Final) es la más aceptada, aunque no la única, no creemos que hoy por hoy sea operativa una división de este tipo, máxime cuando esta nomenclatura parece significar una cosa diferente para cada investigador en la literatura científica.

In this article we do not expect to analyze every single period and all different phases that have
been suggested in describing the evolution of the Andalusian Neolithic. Rather we intend to
discuss specific aspects related to the characterization criteria used in recent proposals of the
tripartite periods. We think this might/will be especially clarifying in relation with the
stratigraphical sequence that we have obtained in La Cueva de los Murciélagos in Zuheros.
Though the most common division is the three phases one (Old, Medium and Final), it is not the
only division. We do not think that it is very useful at this specific moment in time, since this
nomenclature in the scientific literature might actually mean different things for different scholar.

Palabras claves Neolítico, Periodización, Andalucía.

Key words Neolithic, Periodization, Andalucia.

Son numerosos los investigadores que han tratado sobre la periodización del Neolítico en Andalucía desde que se publicaran las excavaciones de Nerja (Pellicer 1963) y Carihuela (Pellicer 1964), pero no está dentro de nuestros objetivos desarrollar en este trabajo un análisis exhaustivo de las propuestas de 
periodización realizadas por los diferentes investigadores desde los comienzos del estudio de las primeras sociedades productoras, sino incidir en algunos aspectos que nos parecen interesantes sobre las distintas fases en que se ha propuesto dividir el Neolítico de la Alta Andalucía recientemente y que de una u otra manera han utilizado argumentos basados en la secuencia de Murciélagos de Zuheros.

Los citados trabajos del Prof. Pellicer en las cuevas de Nerja (1963) y Carihuela (1964) marcaron el comienzo de los estudios sistemáticos sobre el Neolítico en Andalucía, siendo aún obras de obligada consulta y referencia, sobre todo los relativos a la segunda cavidad, habiendo quedado superada la publicación de la primera cueva con la de las excavaciones posteriores (Pellicer y Acosta 1986). En su trabajo sobre Carihuela planteaba, a tenor de los restos contenidos en los distintos niveles de la Cámara G, la existencia de tres fases dentro del Neolítico: Antiguo, definido por la abundancia de la cerámica impresa cardial, que hacía derivar del Levante; Medio, con escasez de dicha especie, aumento de almagras, incisas, impresas no cardiales, grabadas, decoraciones plásticas aplicadas, entre otras; y, por último, Final, representado por el aumento de las cerámicas no decoradas (Pellicer 1964).

La fase Media del Neolítico así establecida y caracterizada cobró especial relevancia tras la publicación de los resultados de là segunda campaña de excavaciones en Murciélagos de Zuheros (Vicent y Muñoz 1973): en ausencia de cardial, se determinó la riqueza material de esta fase en diversos apartados industriales entre los que sobresaldría la cerámica a la almagra. Opinamos que fue la calidad de estas cerámicas, junto con el hecho no menos importante de la constatación en este yacimiento de una economía productora desarrollada y la obtención de algunas de las primeras fechas absolutas, lo que dio lugar a que se considerara, como aún se observa en recientes estados de la cuestión (Martín et alii 1998), como claro exponente del Neolítico Medio. Y todo ello, pese a que nuevas excavaciones y la revisión de las antiguas han puesto de manifiesto que la estratigrafía obtenida en la campaña de 1969, principal pilar sobre el que se sustentaba la caracterización de esta fase -habiéndose propuesto incluso la denominación de "Horizonte Zuheros" en el sentido de neolítico Antiguo y Medio (Arteaga et alii 1993; 1999)-, no resulta en absoluto fiable en lo que se refiere a las comparaciones ergológicas, al constatarse la existencia de cerámica romana -incluso terra sigillata- desde el nivel I al V (Gavilán 1995), cuya presencia queda recogida también en la propia descripción de los materiales en el trabajo publicado por Vicent y Muñoz (1973).

Frente a este criterio de "calidad" de los restos materiales del "Neolítico Medio" de Zuheros, el final de este período es objeto de desacuerdo. Para algunos investigadores viene definido ergológicamente por los conjuntos empobrecidos de la fase Media (Pellicer 1964; Acosta y Pellicer 1990; Gavilán 1989), para otros por la aparición de las cazuelas carenadas (Arribas y Molina 1979), formas que, en sus comienzos, constituyen para Escacena el denominado "Neolítico Atlántico Tardío", diferente al representado por la tradición mediterránea (Escacena et alii 1996); igual adjudicación aunque distinta denominación admiten Pérez Bareas et alii (1999), al indicar que dentro del Neolítico Reciente, el Tardío viene definido por las cazuelas, y el Final por las cerámicas carenadas.

Puesto que no está dentro de nuestros propósitos, como ya hemos indicado, analizar todas y cada una de las periodizaciones y caracterizaciones de las distintas fases que se han propuesto para el Neolítico andaluz, consideramos que con lo mencionado es suficiente para entender que una división en tres fases ha venido siendo, y aún hoy en día es, la más aceptada, aunque no la única. Sin embargo, sí nos interesa atender a determinados aspectos relacionados con la caracterización de las distintas fases de periodización propuestas por Pérez Bareas et alii (1999), que creemos pueden ser objeto de importantes matizaciones.

Así pues, en cuanto al Neolítico Antiguo, aunque se han publicado ya bastantes dataciones y estratigrafías que han motivado un cambio de postura en algunos investigadores, que abogan por un Neolítico Antiguo no cardial en determinados sectores de Andalucía (Acosta y Pellicer 1990), los resultados obtenidos por Pellicer (1964) en Carihuela han arraigado profundamente en numerosos investigadores en lo que respecta a la caracterización de los momentos iniciales del Neolítico a escala regional, puesto que a raíz de la 
publicación de la estratigrafía se sigue considerando casi exclusivamente como Neolítico Antiguo a aquellos conjuntos, incluso de superficie, que arrojan decoraciones cardiales entre sus materiales (Navarrete et alii 1991), obviándose que en la citada cavidad la almagra está presente desde el nivel XV, del Neolítico Antiguo. Incluso, se propone reservar la denominación de "Cultura Andaluza del Neolítico Antiguo" (Pérez Bareas et alii 1999: 488) para la cueva granadina, dentro del "Horizonte Cardial del Neolítico Antiguo" (Ibídem), en contraposición a la de los Murciélagos que, para los citados autores, debe representar el Neolítico Medio, al suponer la presencia de una escasa cantidad de cerámicas impresas a peine.

La periodización que se propone en el citado trabajo para la Alta Andalucía resulta, a nuestro modo de ver, contradictoria con parte de los resultados obtenidos en los últimos años en Murciélagos de Zuheros y en otros yacimientos situados en la provincia de Córdoba, que consideran dentro del ambiente cultural de Andalucía Oriental y pertenecientes exclusivamente al Neolítico Medio, cuando la realidad es que, en lo que respecta a la ocupación de Murciélagos de Zuheros por parte de los primeros grupos productores, en la secuencia se advierte una serie de cambios del material, lógicos a lo largo de un milenio y medio de diferentes ocupaciones (Gavilán et alii 1996), que a la postre es el criterio en que se basan los autores antes citados (Pérez Bareas et alii 1999) para establecer su propuesta de periodización, incurriendo, a nuestro juicio, en una serie de contradicciones, si bien el criterio en sí nos parece válido.

En primer lugar, y rechazando nosotros esa asimilación tajante de Murciélagos de Zuheros con el Neolítico Medio, los autores, basándose en que las principales transformaciones tienen lugar entre el Neolítico Medio y el Tardío -entre las que apuntan la sedentarización y la agregación poblacional-, proponen un Neolítico dividido en una fase Inicial, que sitúan en cronologías no calibradas entre el 5.000 y el 3.500 a.C., y otra Reciente, que se desarrolla entre el 3.500 y el $2.800 / 2.700$ a.C.

Dentro del Neolítico Inicial incluyen tres momentos, para los que proponen una cronología aproximativa y no calibrada: Antiguo Inicial (¿5.000?-4.000 a.C.), Antiguo Avanzado (4.000-3.800 a.C.) y Medio (3.8003.500 a.C.). Teniendo en cuenta que las nuevas fechas de Murciélagos de Zuheros ya han sido publicadas (Gavilán et alii 1996) y que para ellos esta cueva participa de las características de Andalucía Oriental, creemos que deberían haberse tenido presentes en la propuesta cronológica. Por otra parte, no se comprende por qué el Neolítico A de dicho yacimiento (Gavilán et alii 1996), que según la cronología propuesta por los autores está dentro del Neolítico Antiguo Inicial, representa al Neolítico Medio -como se apunta en la nota 2 del trabajo (Pérez Bareas et alii 1999), dando a entender los autores que nosotros mismos realizábamos dicha adscripción-, pues de las once dataciones obtenidas y publicadas en los últimos años, siete están comprendidas entre el 4.480 y el 3.850 a.C.; para ser más exactos, de estas siete fechas, cinco oscilan entre el 4.480 y el 4.240 a.C., es decir, perfectamente dentro de la cronología que proponen para el Neolítico Antiguo Inicial, quedando la restante dentro del Antiguo Avanzado, mientras que sólo contamos con dos -3.710 y 3.626 a.C.- que caen dentro de su Neolítico Medio y otras tantas del Reciente, 3.430 y 3.130 a.C. (Todas las fechas están citadas sin calibrar para posibilitar la comparación con las cronologías propuestas en el citado artículo).

En relación a esto nos preguntamos si resulta conveniente para el avance de la investigación soslayar unos datos -en este caso unas dataciones procedentes de contextos fiables y coherentes entre sí y con las de otras regiones- que no cuadran con una propuesta determinada, de cronología o de periodización.

También observamos una contradicción en lo que se refiere a la caracterización material del Neolítico Antiguo que proponen los autores y la adjudicación por la que optan para el Neolítico A de Murciélagos de Zuheros, teniendo en cuenta que, como hemos indicado, consideran que este yacimiento pertenece culturalmente a Andalucía Oriental. Así, aunque actualmente hay autores que matizan el paradigma cardial como sinónimo de Neolítico Antiguo en Andalucía en general -debido a su escasez, excepción hecha de Carihuela, por el momento-, no es menos cierto que se continúa con la misma concepción con respecto a la caracterización material de dicha fase, puesto que el valor cronocultural de la cardial se substituye 
ahora en algunos yacimientos por las impresas a peine y con matrices dentadas (Pérez Bareas et alii 1999), existentes éstas últimas en numerosas cuevas situadas en la Subbética cordobesa (Gavilán 1989) y en Murciélagos de Zuheros, en la que además son frecuentes las decoraciones plásticas aplicadas, las impresas con distintas matrices y las impresas basculantes -otro de los rasgos culturales que los autores asignan al Neolítico Antiguo Inicial-, que tienen una buena representación en los niveles del Neolítico A, cuya adscripción al Neolítico Antiguo no cardial hemos defendido (Gavilán. 1997).

Al abordar la evolución de la cardial en la Alta Andalucía, se indica (Pérez Bareas et alii 1999) que no desaparece de forma repentina, observándose, como en Levante, la coexistencia con otras impresiones realizadas con matriz dentada. Para los citados autores, esto abre nuevas posibilidades en el cambio de adjudicación cronocultural de determinados conjuntos carentes de contexto que han sido tradicionalmente adscritos al Neolítico Medio, como es el caso de los restos procedentes de la Cueva del Agua de Prado Negro, La Molaina, la Cueva "CV-3", entre otros asentamientos. Con respecto a esto hay dos aspectos que nos resultan igualmente interesantes.

Por una parte, el hecho de acudir a la presencia de cerámicas impresas a peine y mediante una matriz dentada para proponer una ocupación previa a la del Neolítico Medio, de manera que dicha ocupación tendría lugar al final del Antiguo. De este modo, la presencia de ambas matrices decorativas contextualizadas no sólo en Murciélagos de Zuheros -Neolítico A (Gavilán et alii 1996)-, sino en conjuntos de superficie en la Cueva de los Mármoles (Carmona et alii 1999), es otro punto que corrobora, junto con las dataciones de la primera, la ocupación de ambas cuevas a partir de un momento muy temprano del Neolítico.

Por otra parte, resulta sorprendente que los autores sólo hagan uso de materiales de superficie, independientemente en este caso de la fase a que se adscriban, en determinadas zonas geográficas, cuando ellos mismos indican que tales hallazgos resultan improductivos para el avance de la investigación en otras zonas vecinas (Pérez Bareas et alii 1999). Nos preguntamos, en este caso, qué valor tienen las prospecciones y por qué en unos casos los restos de superficie resultan válidos para ser incluidos en una secuencia y en otros no. Por otro lado, esgrimir que la ausencia de cardial puede deberse a recuperaciones no controladas o a prospecciones superficiales, no nos parece adecuado puesto que en determinados yacimientos, conocidos sólo a nivel de materiales de superficie, como Las Majolicas, esta especie se ha constatado.

En lo que se refiere a la caracterización no material de este Neolítico Inicial, los autores indican que el tipo de ocupación es temporal y en directa relación con diferentes estrategias económicas (Pérez Bareas et alii 1999). Ese modelo de ocupación temporal, aunque prolongada y reiterativa, es la que hemos propuesto para los niveles correspondientes al Neolítico A de Murciélagos de Zuheros (Gavilán y Vera 1996; Gavilán et alii 1999; Vera y Gavilán 1999). De este modo, y concluyendo en lo que respecta a este punto, no observamos diferencias sustanciales entre su propuesta de caracterización del Neolítico Inicial y los resultados que ofrecen los primeros niveles neolíticos de Murciélagos de Zuheros, con la salvedad de la ausencia de cardial en la cueva cordobesa, y el hecho de que los productos de alfarería son abundantes desde el comienzo de la ocupación de la cueva por parte de las primeras comunidades productoras. Ciertamente hay estaciones, al aire libre o en cueva, que carecen de cerámicas o son muy escasas, pero opinamos que esto puede deberse, más que a que las verdaderas fases cerámicas tienen lugar durante el Neolítico Medio, al carácter eminentemente estacional de bastantes hábitats y al patrón de asentamiento de estos grupos, caracterizado, al menos en algunos sectores andaluces (Aguayo et alii 1989-90; Gavilán y Vera 1996; 1997), por el establecimiento temporal en amplias cuevas -cuando se trata de conjuntos serranos- y en asentamientos al aire libre, combinado con la ocupación puntual y corta de otras cuevas y estaciones al aire libre para el aprovechamiento de recursos concretos y/o la realización de actividades económicas de subsistencia y no de subsistencia variadas (Gavilán y Vera 1999).

Siguiendo con la secuencia obtenida en Murciélagos de Zuheros, se observa, con respecto al Neolítico A, un cambio en los restos materiales procedentes de los niveles correspondientes al Neolítico B (Gavilán 
et alii 1996), que consideramos perteneciente a un momento avanzado de la anterior fase, y otro con respecto a éste a partir de los niveles del Neolítico C, que marcan el final del neolítico de tradición "mediterránea" en la terminología de Escacena et alii (1996). Estos cambios vienen marcados, en el primer caso, por la mayor abundancia de cerámica a la almagra y decoraciones incisas, al tiempo que escasean impresas basculantes; en el segundo, por el predominio de las no decoradas y la pérdida de calidad en las almagras $y$, en general, de las decoradas, estando ausentes en este yacimiento las formas abiertas tipo cazuela.

Si para establecer clasificaciones hay que acudir a "los rasgos culturales móviles más variables" (Pérez Bareas et alii 1999: 488), los cambios antes indicados, unidos a criterios estratigráficos y a la propia cronología absoluta, nos parecen privilegiados dentro de la generalidad de los contextos andaluces publicados, para haber propuesto una determinada compartimentación tripartita (A, B y C), de una secuencia concreta, que como hemos reiterado no debe traducirse en una división tripartita cultural (Gavilán et alii 1996).

Si nos atenemos a la significancia de probabilidad estadística de las fechas de C-14 de la Estratigrafía de la Cueva Grande de Zuheros, estamos ante tres series distintas de ocupaciones espaciadas temporalmente por unos 350 años en el caso del Neolítico A con respecto al B, y de unos 150 años en el del B con respecto a $\mathrm{C}$, pero dentro de la misma tradición cultural aunque con lógicas diferencias en los restos materiales debido a la separación temporal.

De este modo, si los materiales del Neolítico B de Zuheros podrían concordar bien con parte de las características propuestas para el Neolítico Medio de los autores (Ibídem)-incluido en el Neolítico Inicial-, la cronología no iría en consonancia, y al contrario, nuestra fase $\mathrm{C}$ que, según la periodización que proponen, habría que considerar por sus fechas como Neolítico Tardío, no concordaría con la mayor parte de los rasgos culturales seleccionados.

En cuanto al Neolítico Reciente, como ya hemos apuntado en otro trabajo (Gavilán 1997), se define, dependiendo de los investigadores, bien por los conjuntos empobrecidos de la fase Media, bien por la presencia de formas abiertas. Esta es la opción defendida por Escacena (Escacena et alii 1996), Pérez Bareas (Pérez Bareas et alii 1999), entre otros.

Si tenemos en cuenta la propuesta cronológica defendida por los citados autores (Pérez Bareas et alii 1999), ya hemos observado una correspondencia cronológica, que no industrial ni cultural, entre el Neolítico C de Zuheros -entre el 3.430 y el 3.130 a.C.-y el Neolítico Reciente Tardío-3.500 al 3.100 a.C. Al respecto, debe tenerse en cuenta que otros yacimientos neolíticos andaluces han proporcionado dataciones en consonancia con las de Zuheros, caso de Nerja, que ofrecen además unos conjuntos industriales igualmente parejos y en los que no se constata, o lo hace de forma francamente escasa, la existencia de formas abiertas tipo cazuela, vasijas que definen el Neolítico Reciente, dividido entre Tardío y Final, de Pérez Bareas (Ibídem).

Realmente, en las secuencias amplias de cuevas que cuentan con niveles adjudicados por tal ocual autor a la fase Media del período, no se advierte una evolución en la cerámica hacia las formas abiertas, sino que éstas se documentan en una fecha aún no precisada con exactitud pero que se sitúa en un momento ligeramente anterior al 3.000 a.C. en fechas no calibradas. Es más, en bastantes zonas en las que se ha documentado el Neolítico de tradición mediterránea, las cazuelas carenadas suelen ser francamente escasas, cuando no están ausentes, como ocurre, dentro de la Alta Andalucía, en la Subbética cordobesa; por el contrario, en sectores próximos, como Martos (Lizcano et alii 1991-92), Montefrío (Afonso et alii 1996) y la propia campiña de la zona de Castro del Río (Carrilero y Martínez 1985), en la provincia de Córdoba, estas formas cerámicas están bien representadas.

Esta correspondencia entre distintos yacimientos neolíticos andaluces que han aportado conjuntos industriales de tradición mediterránea, por un lado, y la existencia de otros asentamientos de semejante cronología, generalmente ahora al aire libre, con formas cerámicas tipo cazuelas, y otros elementos no menos importantes, ausentes en el primer tipo de yacimiento, por otro, puede estar indicando, como defiende 
Escacena (Escacena et alii 1996), dos tradiciones culturales diferentes y en cierta medida sincrónicas que cubren parte de la fase más reciente del Neolítico.

A tenor de lo expuesto, consideramos que el panorama es más complejo de lo que se pretende y que la reciente periodización y clasificación de rasgos culturales propuestas para el Neolítico de la Alta Andalucía resulta excesivamente rígida para una zona tan amplia y variada, sobre todo teniendo en cuenta que determinadas secuencias estratigráficas no se ajustan al modelo. A nuestro modo de ver, cualquier trabajo de este tipo debe partir del estudio de áreas más restringidas para ser contrastadas posteriormente con otros sectores. De lo contrario, yacimientos que han proporcionado una amplia secuencia en la que se advierten cambios materiales no tienen cabida en dichas clasificaciones, como es el caso de Murciélagos de Zuheros, considerado por determinados autores (Pérez Bareas et alii 1999) como Neolítico Medio exclusivamente.

En este sentido finalizamos indicando que, según la estricta propuesta de clasificación y periodización de los autores (Ibídem), dicha fase abarca sólo unos 300 años, pero en Murciélagos de Zuheros, según las fechas, duraría 1.350 años, lo cual refleja la inoperancia de aplicar encorsetamientos ya sean de tres o cinco fases a la realidad arqueológica actual del Neolítico andaluz.

\section{BIBLIOGRAFÍA}

ACOSTA, P. y PELLICER, M. (1990): La Cueva de la Dehesilla (Jerez de la Frontera). Las primeras civilizaciones productoras en Andalucía Occidental. Jerez de la Frontera.

AFONSO, J.A.; MOLINA, F. y CÁMARA, J.A. (1996): "Espacio y tiempo. La secuencia en Los Castillejos de Las Peñas de Los Gitanos (Montefrío, Granada)", I Congrés del Neolitic a la Península Ibérica. Formació e implantació de les comunitats agrícoles (Gavá-Bellaterra, 1995), Actes Vol, 1.(J. Bosch, M. Molist, Orgs.), Rubricatum 1, 1: 297-304, Gavá.

AGUAYO,P; MARTÍNEZ, G. y MORENO,F. (1989-90): “Articulación de los sistemas de hábitats Neolítico y Eneolítico en función de la explotación de los recursos naturales en la Depresión de Ronda", C.P.U.Gr 14-15: 67-84.

ARRIBAS, A. y MOLINA, F. (1979): El pobladode <Los Castillejos > en Las Peñas de los Gitanos (Montefrío, Granada). Campaña de excavaciones de 1971. El corte número 1. C.P.U.Gr. Serie Monográfica 3.

ARTEAGA, O.; RAMOS; J.; ROOS, A.M. y NOCETE, F. (1991): "Balance a medio plazo del Proyecto Porcuna>. Campaña de 1991", Anuario Arqueológico de Andalucía, II Actividades Sistemáticas: 295-301.

ARTEAGA, O. y CRUZ-AUÑÓN, R. (1995): "El asentamiento neolítico al aire libre de <Los Álamos> (Fuentes de Andalucía, Sevilla). Excavación de urgencia de 1995", Anuario Arqueológico de Andalucía, III Actividades de Urgencia: 559-566.

CARMONA; R.; MORENO, A.; VERA, J.C.; LUNA, D.; GAVILÁN, B. y MOLINA, A. (1999): "La Cueva de los Mármoles (Priego de Córdoba): Análisis de resultados de una prospección arqueológica superficial”, Antiquitas 10: 5-25. Priego de Córdoba.

CARRILERO, M y. MARTÍNEZ, G. (1985): "El yacimiento de Guta (Castro del Río) y la Prehistoria Reciente de la Campiña Cordobesa", C.P.U.Gr. 10: 187-223.

ESCACENA, J.L.; RODRÍGUEZ DEZULOAGA, M. y LADRÓNDE GUEVARA, I. (1996): Guadalquivir salobre. Elaboración prehistórica de sal marina en las antiguas bocas del río. Confederación Hidrográfica del Guadalquivir, Sevilla. 
GAVILÁN, B. (1989): El Neolítico en el Sur de Córdoba. Análisis sistemático de las primeras culturas productoras. Anexos de Estudio de Prehistoria Cordobesa. 2 vols. Univ. de Córdoba.

- (1995): "Estudio de materiales arqueológicos procedentes de la Cueva de los Murciélagos de Zuheros: Revisión de las campañas de 1962, 1969 y materiales de superficie", Anuario Arqueológico de Andalucía 1992, II, Actividades Sistemáticas: 103-106.

- (1997): "Reflexiones sobre el Neolítico Andaluz", Spal 6: 23-33. Sevilla.

GAVILÁN, B. y VERA, J.C. (1996): "Estaciones neolíticas al aire libre en el Sureste de la provincia de Córdoba", Antiquitas 7: 5-18.

(1997): "Nuevos datos sobre los patrones de poblamiento neolítico y calcolítico al aire libre en el piedemonte de las Sierras Subbéticas", Antiquitas 8: 5-22.

- (1999): "El patrón de asentamiento al aire libre de las primeras comunidades productoras de Andalucía central en el marco del poblamiento neolítico del Sur de la Península Ibérica", XXIV Congreso Nacional de Arqueología (Cartagena, 1997), Vol. 2, El mundo indígena del Neolítico al Bronce: 31-36.

GAVILÁN, B.; VERA, J.C.; PEÑA, L. y MAS, M. (1996): "El V y el IV Milenios en Andalucía Central: La Cueva de los Murciélagos de Zuheros (Córdoba). Recientes aportaciones." I Congrés del Neolitic a la Península Ibérica. Formació e implantació de les comunitats agrícoles (Gavá-Bellatera, 1995). Actes. Vol. I. (J. Bosch, M. Molist, Orgs.), Rubricatum, 1, 1: 323-327, Gavá.

GAVILÁN, B.; VERA, J.C.; MORENO, A. y MAS, M. (1999): "Cazadores-recolectores y primeros productores en el Macizo de Cabra (Córdoba)" en L. Pallí y C. Roqué [Eds.] Avances en el estudio del Cuaternario español. X Reunión Nacional de Cuaternario (Gerona, 1999): 259-263.

LIZCANO, R.; CÁMARA, J.A.; RIQUELME, J.A.; CABAÑATE, M.L.; SÁNCHEZ, A. y AFONSO, J.A. (1991-92): "El Polideportivo de Martos. Estrategias económicas y símbolos de cohesión en un asentamiento del Neolítico Final del Alto Guadalquivir", C.P.U.Gr. 16-17: 5-101.

MARTÍN, D.; CAMALICH, M.D. y GONZÁLEZ, P. (1998): "L'Andalousie”, Atlas du Néolithique européen. Vol. 2 B. L'Europe occidentale. E.R.A.U.L., 46: 871-933.

NAVARRETE, M.S.; CAPEL, J.; LINARES, J.; HUERTAS, F. y REYES, E. (1991): Cerámicas Neolíticas de la provincia de Granada. Materias primas y técnicas de manufacturación. Monográfica Arte y Arqueología 9. Granada.

PELLICER, M. (1963): Estratigrafía prehistórica de la Cueva de Nerja, $1^{a}$. campaña, 1959. E.A.E. 16.

- (1964): El Neolítico y el Bronce de la Cueva de la Carihuela de Pínar (Granada). T.P. XV.

PELLICER, M. y ACOSTA,P.(1986): La Prehistoria de la Cueva de Nerja (Málaga). Neolítico y Calcolítico. Málaga.

PÉREZ, C.; AFONSO, J.A.; CÁMARA, J.A.; CONTRERAS, F. y LIZCANO, R. (1999): “Clasificación cultural, periodización y problemas de compartimentación en el Neolítico de la Alta Andalucía", II Congrés del Neolitic a la Península Ibérica (Valencia, 1999)) Actes. (J. Bernabeu, T. Orozco, Eds.). Sagvntvm, Extra-2: 485-492.

VERA, J.C. y GAVILÁN, B. (1999): “Organización interna y usos del espacio en la Cueva de los Murciélagos de Zuheros (Córdoba)", Sagvntum Extra-2, Actes del II Congrés del Neolític a la Península Ibérica (Valencia, 1999): 229-234.

VICENT, A.M. y MUÑOZ, A.M.(1973): Segunda campaña de excavaciones. La Cueva de los Murciélagos, Zuheros (Córdoba), 1969. E.A.E. 77. 